\title{
Automatic generation of vertices for the Schrödinger functional
}

\author{
Shinji Takeda* \\ Humboldt Universitaet zu Berlin \\ E-mail: takeda@physik.hu-berlin.de

\section{Ulli Wolff} \\ Humboldt Universitaet zu Berlin \\ E-mail: uwolffephysik.hu-berlin.de
}

\begin{abstract}
We present a multiplication algorithm to recursively construct vertices for the Schrödinger functional in the abelian background field case. The algorithm is suited for automatic perturbative calculations with a variety of actions. As first applications, we derive ratios of the lambda parameters between the lattice scheme (improved gauge actions including six link loops) and the $\overline{\mathrm{MS}}$ scheme, and one-loop results for the Schrödinger functional coupling with a lattice $T=L \pm a$, which is motivated by considering staggered fermions.
\end{abstract}

The XXV International Symposium on Lattice Field Theory

July 30 - August 42007

Regensburg, Germany

${ }^{*}$ Speaker. 


\section{Introduction}

It is well known that in lattice gauge theory the vertices are quite complicated especially for the pure gluon sector. This is because, on the lattice, we are preserving gauge invariance at finite cutoff and the lattice itself breaks the Lorentz symmetry explicitly. This is the main difficulty of lattice perturbation theory. To reduce the risk of errors and to alleviate the tedious task of deriving the vertices, it is desirable to have an automatic method. A first attempt was made by Lüscher and Weisz about twenty yeas ago [1]. They worked in momentum space and performed some calculations by using their algorithm which was restricted to closed loops sufficient for pure gauge theory.

Recently a new algorithm, that we call bottom up algorithm, was proposed by Hart et. al.[2]. A crucial point in this generalization is that it can deal with any parallel transporter, not only with closed loops. This allows to also include a fermion action or even a smeared HQET action. A relevant assumption for this algorithm has been translation invariance. Our main concern in this note is to extend the bottom up algorithm to the Schrödinger functional (SF)[3] where this invariance is broken for the time direction. Before going to the extension we first summarize the position space version of the algorithm on the usual translation invariant lattice in the next section.

\section{Bottom up Algorithm}

In Ref.[2], the authors explain the algorithm in momentum space but here we will move to coordinate space. In the following we denote the antihermitean gauge fluctuation field by $q_{\mu}(x)$, and the link variable (still without background field) by $U(x, \mu)=\exp \left(g_{0} q_{\mu}(x)\right)$.

A first important point for the automatic operation is how to represent the vertices in a program. We consider the parallel transporter along a curve $\mathscr{L}$ on the lattice. The $r$ 'th order coefficient in the Taylor expansion $P_{r}$ of the parallel transporter $P[\mathscr{L} ; q]$ is written as

$$
P[\mathscr{L} ; q]=\sum_{r=0}^{\infty} \frac{g_{0}^{r}}{r !} P_{r}[\mathscr{L} ; q], \quad P_{r}=\sum_{a_{1}, \ldots, a_{r}} \mathscr{C}_{a_{1} \cdots a_{r}} \sum_{k=1}^{N_{r}} q_{a_{1}}^{\alpha_{k}^{1}} \cdots q_{a_{r}}^{\alpha_{k}^{r}} f_{k}
$$

where $\alpha=(\mu, x)$ is a combined index labelling links, and $a$ is color. We call $\mathscr{C}_{a_{1} \cdots a_{r}}=T_{a_{1}} \cdots T_{a_{r}}$ a color factor, and $f_{k}$ an amplitude. The latter corresponds to a value of a reduced vertex with index $\left(\alpha_{k}^{1}, \cdots, \alpha_{k}^{r}\right)$. The reduced vertex here means a vertex without the color factor. The information about the reduced vertex of order $r$ is now encoded in a list consisting of $N_{r}$ lines

$$
L_{k}^{(r)}=\left(\alpha_{k}^{1}, \cdots, \alpha_{k}^{r} ; f_{k}\right)
$$

which holds the index structures and amplitudes of the corresponding reduced vertex. The list $L^{(r)}=\left\{L_{k}^{(r)} \mid k=1, . ., N_{r}\right\}$ corresponds to the all nonzero elements of the reduced vertex of order $r$. It will be constructed recursively by the multiplication algorithm to be given below. Note that in eq.(2.1) the color factor is located outside of the $k$-summation, because it is independent of the shape of the transporter. However, for the SF this nice structure is rendered more complicated by the background field as we will see in the next section. 
As an explicit example for a list, let us look at the case of the single gauge link variable. From the expanded form we can obtain a list $L^{(r)}$ of the link variable,

$$
U(x, \mu)=\sum_{r=0}^{\infty} \frac{g_{0}^{r}}{r !} \sum_{a_{1} \cdots a_{r}} \mathscr{C}_{a_{1} \cdots a_{r}} q_{a_{1}}^{\alpha} \cdots q_{a_{r}}^{\alpha}, \quad L^{(r)}=(\underbrace{\alpha, \cdots, \alpha}_{r \text { elements }} ; 1), \quad \alpha=(\mu, x) .
$$

It consists of only one line $\left(N_{r}=1\right)$ and is the elementary building block of the algorithm, that is, something like an initial condition.

So far we have defined the fundamental elements on which the algorithm operates. Next we consider the case that a parallel transporter $P$ is composed from $P^{\prime}$ and $P^{\prime \prime}, P=P^{\prime} P^{\prime \prime}$ and want to obtain the vertices of $P$ from those of $P^{\prime}$ and $P^{\prime \prime}$. In other words, the problem is how to obtain a set of lists of $P$ (up to a certain order), from those of $P^{\prime}$ and $P^{\prime \prime},\left\{L_{i}\right\}_{P^{\prime}} \times\left\{L_{j}\right\}_{P^{\prime \prime}} \longrightarrow\left\{L_{k}\right\}_{P}$. Since any parallel transporter is composed of elementary one-link variables, by repeating the procedure, we can obtain the lists for arbitrary parallel transporters. This is the origin of the name 'bottom up'. The algorithm can be easily understood by looking at an actual multiplication of the coefficients of the Taylor expansion. The coefficient of $P$ with order $r, P_{r}$, is expressed by those of $P^{\prime}$ and $P^{\prime \prime}$ as

$$
\begin{aligned}
P_{r} & =\sum_{s=0}^{r} \frac{r !}{s !(r-s) !} P_{s}^{\prime} P_{r-s}^{\prime \prime} \\
& =\sum_{a_{1} \ldots a_{r}} \mathscr{C}_{a_{1} \ldots a_{r}} \sum_{s=0}^{r} \frac{r !}{s !(r-s) !} \sum_{i=1}^{N_{s}^{\prime}} \sum_{j=1}^{N_{r-s}^{\prime \prime}} q_{a_{1}}^{\alpha_{i}^{1}} \ldots q_{a_{s}}^{\alpha_{i}^{s}} q_{a_{s+1}}^{\alpha_{j}^{1}} \ldots q_{a_{r}}^{\alpha_{r-s}^{r-s}} f_{i}^{\prime} f_{j}^{\prime \prime} \\
& =\sum_{a_{1} \ldots a_{r}} \mathscr{C}_{a_{1} \ldots a_{r}} \sum_{k=1}^{N_{r}} q_{a_{1}}^{\alpha_{k}^{1}} \ldots q_{a_{r}}^{\alpha_{k}^{r}} f_{k} .
\end{aligned}
$$

After the second equal-sign we inserted the explicit form of the coefficients, and use the fact that the color factor is independent of the shape of the parallel transporter. In the last step we combine the three summations into that over $k$. More precisely, we did a relabelling of the indices, and rewrote the amplitude factor. Finally the resulting list of $P, L_{k}$ is created by putting the new label structure and the new amplitude. The algorithm is summarized as

- Relabeling: $\left\{\alpha_{i}^{1}, \cdots, \alpha_{i}^{s}, \alpha_{j}^{1}, \cdots, \alpha_{j}^{r-s}\right\} \longrightarrow\left\{\alpha_{k}^{1}, \cdots, \alpha_{k}^{s}, \alpha_{k}^{s+1}, \cdots, \alpha_{k}^{r}\right\}$

- Amplitude part: $\frac{r !}{s !(r-s) !} f_{i}^{\prime} f_{j}^{\prime \prime} \longrightarrow f_{k}$

- Creating list: $L_{i}^{\prime} \times L_{j}^{\prime \prime} \longrightarrow L_{k}=\left(\alpha_{k}^{1}, \cdots, \alpha_{k}^{r} ; f_{k}\right)$

This procedure should be carried out for $0 \leq s \leq r, 1 \leq i \leq N_{s}^{\prime}$ and $1 \leq j \leq N_{r-s}^{\prime \prime}$ if order $r$ is desired. The algorithm has been implemented in the python script language, which is good at dealing with the complicated list operation. In this way, one can obtain the vertices for any parallel transporter.

\section{Extension to the Schrödinger Functional}

The essential new ingredient in the SF is the presence of the non-vanishing back ground field. It is induced by non trivial Dirichlet boundary conditions in the time direction. In this case, the link variable is expressed by the background field and the fluctuation field $q_{\mu}$,

$$
U(x, \mu)=V(x, \mu) e^{g_{0} q_{\mu}(x)} .
$$


The main difficulty due to the presence of the back ground field occurs in the color factor. In the color factor, the back ground field can appear "randomly" between SU(3) generators, for example

$$
\mathscr{C}_{a_{1} a_{2} a_{3} \cdots}=T_{a_{1}} T_{a_{2}} T_{a_{3}} \cdots \longrightarrow T_{a_{1}} V T_{a_{2}} V^{-1} T_{a_{3}} \cdots, \quad \text { on SF }
$$

where arguments of $V$ 's and powers $\left(V\right.$ or $\left.V^{-1}\right)$ depend on the location of the links and its orientation. At first glance, it seems difficult to deal with it in a systematic way. Furthermore, since the background field depends on the Lorentz and position indices, the color factor is located inside of the sum over $i$ and does not factorize anymore,

$$
P_{r}=\sum_{a_{1}, \ldots, a_{r}} \mathscr{C}_{a_{1} \cdots a_{r}} \sum_{k=1}^{N_{r}} q_{a_{1}}^{\alpha_{k}^{1}} \cdots q_{a_{r}}^{\alpha_{k}^{r}} f_{k} \longrightarrow \sum_{a_{1}, \ldots, a_{r}} \sum_{k=1}^{N_{r}} \mathscr{C}_{a_{1} \cdots a_{r}}^{k} q_{a_{1}}^{\alpha_{k}^{1}} \cdots q_{a_{r}}^{\alpha_{k}^{r}} f_{k}, \quad \text { on SF. }
$$

One can solve the problem however for the restricted class of background fields that have mainly been used in applications of the SF [3], $V(x, 0)=1, V(x, k)=V\left(x_{0}\right)$. In addition, we take the background field to be abelian, given by diagonal color matrices. This means that the generators in the $V\left(x_{0}\right)$ are written as linear combinations of the elements of the Cartan sub-algebra $H_{j}$, that is, $V\left(x_{0}\right)=e^{i \Sigma_{j} h_{j}\left(x_{0}\right) H_{j}}$ with coefficients $h_{j}\left(x_{0}\right)$. Now we find the nice equation, $V\left(x_{0}\right) I_{a} V^{-1}\left(x_{0}\right)=$ $I_{a} e^{i \phi_{a}\left(x_{0}\right)}$, and the $I_{a}$ (Cartan basis) do not mix with other basis elements. This is because, in the adjoint representation, an element of the Cartan basis is an eigenstate of the Cartan generator, $\left[H_{j}, I_{a}\right]=\mu_{j a} I_{a}$. The eigenvalue $\mu$ is a root, and the phase is a linear combination of the roots and $h_{j}\left(x_{0}\right), \phi_{a}\left(x_{0}\right)=\sum_{j} \mu_{j a} h_{j}\left(x_{0}\right)$. Another property of the standard background fields that is relevant here is that the back ground field and the phase has a simple dependence on time, $V\left(x_{0}+\Delta t\right)=$ $V\left(x_{0}\right) \exp (i \Delta t \mathscr{E}), \phi_{a}\left(x_{0}+\Delta t\right)=\phi_{a}\left(x_{0}\right)+\Delta t \psi_{a}$, where $\mathscr{E}$ is the color electric field. This however needs to be relaxed later, see in next section.

Our main finding is that by making use of the above properties of the background field any color factor of order $r$ can be written as

$$
\mathscr{C}_{a_{1} \cdots a_{r}}^{k}\left(x_{0}\right)=\underbrace{\left[I_{a_{1}} \cdots I_{a_{r}} V^{A_{k}}\left(x_{0}\right) e^{i \mathscr{E} B_{k}}\right]}_{3 \times 3 \text { matrix }} \underbrace{e^{\frac{i}{2} \sum_{u=1}^{r}\left(\psi_{a_{u}} C_{k}^{(u)}+\phi_{a_{u}}\left(x_{0}\right) D_{k}^{(u)}\right)},}_{U(1) \text { phase factor }}
$$

where the background field has been moved to the right in the $3 \times 3$ matrix part. Actually we can show by induction that $A$ and $B$ are single component integer, and $C$ and $D$ are $r$ component integer valued vectors. In the expression, the information of the lattice size and the back ground field are encoded in $V, \mathscr{E}, \phi$ and $\psi$. The lists are independent of these values, only $x_{0}, A, B, C, D$ are required. The former are only needed when implementing the vertex in a diagram calculation program at a second stage. Note that we need $x_{0}$, since there is no translation symmetry for the time direction. We choose $x_{0}$ as the time component of the position of the left most link variable in the parallel transporter. The benefit of the expression is that we can separate the information about the list $(A, B, C, D)$ and the lattice size and the back ground field. Therefore, we can do a symbolic list operation, independently of the details of the lattice and the background field.

We have obtained a manageable expression for the color factor. Next we formulate the multiplication for this structure of integer lists $\left(x_{0}, A, B, C, D\right)$. From an actual multiplication of the color factors, we found the algorithm to get a list of color factor $\mathscr{C}$ (order $r$ ) from those of $\mathscr{C}^{\prime}$ (order $s$ ) 
and $\mathscr{C}^{\prime \prime}($ order $r-s),\left(x_{0}, A, B, C, D\right) \longleftarrow\left(x_{0}^{\prime}, A^{\prime}, B^{\prime}, C^{\prime}, D^{\prime}\right) \times\left(x_{0}^{\prime \prime}, A^{\prime \prime}, B^{\prime \prime}, C^{\prime \prime}, D^{\prime \prime}\right)$,

$$
\begin{aligned}
& x_{0} \longleftarrow x_{0}^{\prime}, \\
& A \longleftarrow A^{\prime}+A^{\prime \prime}, \\
& B \longleftarrow B^{\prime}+B^{\prime \prime}+\Delta t A^{\prime \prime}, \\
& C \longleftarrow \underbrace{\underbrace{C_{\text {elements }}^{\prime}}_{(1), \cdots, C_{(s)}^{\prime}}, \underbrace{C_{(1)}^{\prime \prime}+2 B^{\prime}+\Delta t D_{(1)}^{\prime \prime}, \cdots, C_{(r-s)}^{\prime \prime}+2 B^{\prime}+\Delta t D_{(r-s)}^{\prime \prime}}_{r-s \text { elements }})}_{r \text { elements }}, \\
& D \longleftarrow \underbrace{(\underbrace{D_{(1)}^{\prime}, \cdots, D_{(s)}^{\prime}}_{s \text { elements }}, \underbrace{D_{(1)}^{\prime \prime}+2 A^{\prime}, \cdots, D_{(r-s)}^{\prime \prime}+2 A^{\prime}}_{r-s \text { elements }})}_{r \text { elements }},
\end{aligned}
$$

where $\Delta t=x_{0}^{\prime \prime}-x_{0}^{\prime}$. It turns out that the resulting $A$ and $B$ remain single component integer. On the other hand, the resulting $C$ and $D$ are given by combinations of single prime and double prime objects with some additional terms. Since $A, B, C, D$ are all integer value and this operation is simple, the algorithm is suited for a symbolic operation and easily implemented in python script language. As a new ingredient in the implementation, we have to add the new components $x_{0}, A, B, C, D$ to the earlier list structure,

$$
L_{k}^{(r)}=\left(\alpha_{k}^{1}, \cdots, \alpha_{k}^{r}, x_{0}, A_{k}, B_{k}, C_{k}, D_{k} ; f_{k}\right)
$$

Even in the SF with non-trivial color factor, the algorithm maintains a closed multiplication structure, therefore it is applicable for any parallel transporter.

To confirm and check the algorithm, we perform a one-loop calculation of the SF coupling. By calculating this quantity, we can check the two-point vertex, that is, inverse propagator. We investigate not only the plaquette gauge action considered before [3], but also the improved gauge actions including the rectangular loop [4] and get consistent results. Furthermore, we compared with the hand derived three point vertex of the plaquette gauge action, available from a private note of Peter Weisz and confirmed consistency. To get further confidence in the implementation of our algorithm, we have to check the four-point vertex. To do so we need to do a two-loop calculation of the SF coupling [5, 6], and this will be reported in the future.

\section{Application I: $L=T \pm a$ lattice}

As a simple novel application of our algorithm, we perform a one-loop computation of the SF coupling on lattices with $L=T+s a$ with $s= \pm 1$. Such lattices are motivated by considering the SF with staggered fermions. Due to the Dirichlet boundary in the time direction, we have to set $T$ to be odd. Here, we will discuss only the gauge part on the lattice, not the staggered fermion part. More details about the latter are given in the contribution of P. Perez Rubio and S. Sint to this conference.

When one takes the continuum limit in the standard SF, one sets the $T / L=1$. The fact that we here have to set $(T+s a) / L=1$ when taking the continuum limit in the tree-level $O(a)$ improved theory causes some change to the solution of the equation of motion, that is, the background field. It 

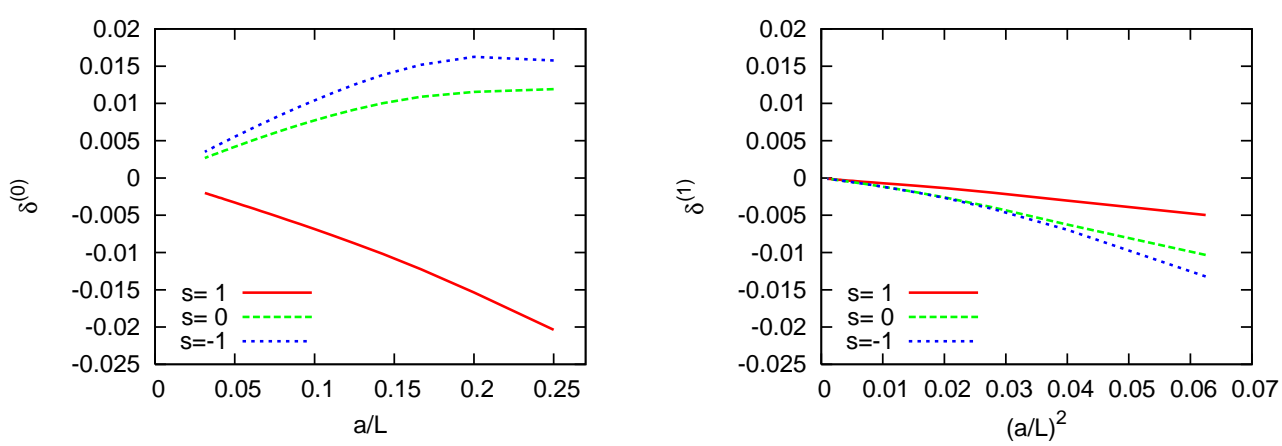

Figure 1: One-loop relative deviation as a function of $a / L$. The left(right) panel shows tree(one-loop) level $O(a)$ improved results

is modified by lattice artifacts as compared to the standard one, and has to be extracted numerically from the equation of motion. The time dependence of the background field turns out not to be strictly linear anymore. Therefore we have to extend our algorithm to apply for an arbitrary time dependent phase $\phi\left(x_{0}\right)$. For this purpose, we have derived another variant algorithm for the color factor which is similar to eq.(3.9), and it will be shown in more detail in a future publication.

In Figure 1, we show the resulting relative deviation of the step scaling function to one-loop order, $\delta_{1}^{(k)}(a / L)$, given by

$$
\begin{aligned}
\delta(u, a / L) & =\frac{\Sigma(u, a / L)-\sigma(u)}{\sigma(u)}=\delta_{1}^{(k)}(a / L) u+O\left(u^{2}\right), \\
\sigma(u) & =\bar{g}^{2}(2 L), \quad u=\bar{g}^{2}(L),
\end{aligned}
$$

where $k=0(k=1)$ is the tree (one-loop)level $\mathrm{O}(a)$ improved case. In the plots, for comparison, we also include those of $s=0$ where $L$ and $T$ are the same. As a result, we observe that all three cases have similar absolute size of the lattice artifacts.

\section{Application II: $\Lambda$ parameter for improved gauge actions}

As a next application, we apply the algorithm to the improved gauge actions including six link loops (not only for the rectangular type but also for the chair and 3-dimensional type actions). The loops are shown in Figure 2 with weight factors, $c_{0}, c_{1}$ etc. and the weights are normalized by $c_{0}+8 c_{1}+16 c_{2}+8 c_{3}=1$. We perform the one-loop computation of the SF coupling for the improved gauge actions and extract information about a ratio of lambda parameters for the pure SU(3) gauge theory between the lattice scheme (the various gauge actions) and the SF scheme, $\Lambda_{\mathrm{Lat}} / \Lambda_{\mathrm{SF}}$. By combining the result of $\Lambda_{\mathrm{SF}} / \Lambda_{\overline{\mathrm{MS}}}$ in [6] for $N=3$ and $N_{\mathrm{f}}=0$, we summarize the values of $\Lambda_{\mathrm{Lat}} / \Lambda_{\overline{\mathrm{MS}}}=\Lambda_{\mathrm{Lat}} / \Lambda_{\mathrm{SF}} \cdot \Lambda_{\mathrm{SF}} / \Lambda_{\overline{\mathrm{MS}}}$ in Table 1 . We observe rough consistency with old results.

We thank the Deutsche Forschungsgemeinschaft (DFG) for support in the framework of SFB Transregio 9. 


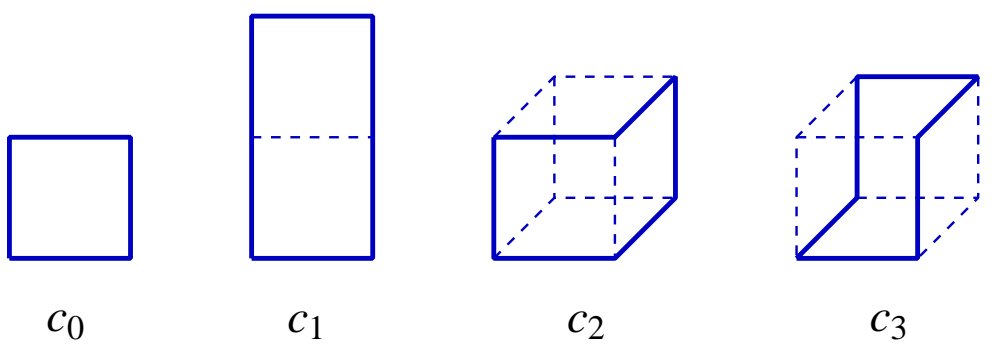

Figure 2: Loops with weight factors $c_{0,1,2,3}$ which contribute to the improved gauge action.

\begin{tabular}{|c|ccc|c|}
\hline \hline Action & $c_{1}$ & $c_{2}$ & $c_{3}$ & $\Lambda_{\text {Lat }} / \Lambda_{\overline{\mathrm{MS}}}$ \\
\hline Plaquette & 0 & 0 & 0 & $0.0347109675 \ldots[7]$ \\
\hline Wilson RG & -0.252 & 0 & -0.170 & $2.34086(2)$ \\
\hline Iwasaki & -0.331 & 0 & 0 & $2.12455(5)$ \\
\hline DBW2 & -1.40686 & 0 & 0 & $44.21(2)$ \\
\hline Symanzik & $-1 / 12$ & 0 & 0 & $0.1836938(4)$ \\
\hline Symanzik II & $-1 / 12$ & $1 / 16$ & $-1 / 16$ & $0.1782883(4)$ \\
\hline no name & $-1 / 12$ & -0.1 & 0.1 & $0.1673674(8)$ \\
\hline \hline
\end{tabular}

Table 1: We show our results of $\Lambda_{\mathrm{Lat}} / \Lambda_{\overline{\mathrm{MS}}}$ in SU(3) gauge theory for various gauge actions, except for the plaquette gauge action ref. [7] which is given here for completeness. Our values are roughly consistent with old results $[8,9,10,11,12,13]$, but in ref.[13] the authors do not show error estimates therefore, it is hard to compare. After the completion of this work, new results for several gauge actions have appeared in ref. [14].

\section{References}

[1] M. Lüscher and P. Weisz, Nucl. Phys. B266 (1986) 309.

[2] A. Hart, G. M. von Hippel, R. R. Horgan and L. C. Storoni, J. Comput. Phys. 209 (2005) 340.

[3] M. Lüscher, R. Sommer, P. Weisz and U. Wolff, Nucl. Phys. B413 (1994) 481.

[4] S. Takeda, S. Aoki and K. Ide, Phys. Rev. D68 (2003) 014505.

[5] A. Bode, U. Wolff and P. Weisz, Nucl. Phys. B540 (1999) 491.

[6] A. Bode, P. Weisz and U. Wolff, Nucl. Phys. B576 (2000) 517.

[7] M. Lüscher and P. Weisz, Phys. Lett. B349 (1995) 165.

[8] P. Weisz and R. Wohlert, Nucl. Phys. B236 (1984) 397.

[9] Y. Iwasaki and S. Sakai, Nucl. Phys. B248 (1984) 441.

[10] Y. Iwasaki and T. Yoshie, Phys. Lett. B143 (1984) 449.

[11] A. Ukawa and S.-K. Yang, Phys. Lett. B137 (1984) 201.

[12] W. Bernreuther, W. Wetzel and R. Wohlert, Phys. Lett. B142 (1984) 407.

[13] S. Sakai, T. Saito and A. Nakamura, Nucl. Phys. $B 584$ (2000) 528.

[14] A. Skouroupathis and H. Panagopoulos, hep-lat/0709.3239 